\title{
Modeling a Bandwidth of a Two-Level Independent Quantum Laser System States
}

\section{Cliff Orori Mosiori $^{1}$}

${ }^{1}$ Technical University of Mombasa

P. 0. Box 90420-80100, Mombasa, Kenya

DOI: $10.22178 /$ pos.29-2

LCC Subject Category:

TP155-156, QC450-467, QD450-801

Received 11.11.2017

Accepted 10.12.2017

Published online 18.12.2017

Corresponding Author: cliffmosiori@gmail.com

(C) 2017 The Author. This article is licensed under a Creative Commons Attribution 4.0 License
Abstract. An electromagnetic radiation is basically considered to be a transverse wave propagating through an accessible media whether it is an optical fibre or a thin film depending on its propagating conditions. In analyzing its propagation, utilized specific cross-section areas have become an important base of understanding its extinction parameters. The term cross-section in this work applies only to boundary conditions within which particles interact within electromagnetic spectra through absorption or scattering. In such small volume contextual framework, nanotechnology reconsiders scattering cross-section coefficients. In this work, two different degenerate states were modeled and analyzed using a developed model of a laser cavity containing a media of length, $L$, with a gain of, $k$, per unit length which were mirrored to represent the upper and a lower level manifolds inhomogeneous broadening Stark levels respectively. The model developed created an impression that a laser medium is a coherent ensemble of particles or atoms whose bandwidth depends on how a laser is constructed.

Keywords: Einstein coefficient; spontaneous emission; spectral line profile function; small-signal gain coefficient; population inversion; laser cavity.

\section{INTRODUCTION}

On a simplified basic fundamental scale, any EM wave propagating on an accessible media gain or lose energy as a result excitation of quantum confinement states at certain regions and thus a particle's specific cross-section becomes an important base for analyzing its extinction parameters $[1,16,20]$. Cross-sections here use boundary conditions within which absorbing or scattering a radiation takes place. Such cross sections are different for specific geometric cross sectional area of the particle making the overall area be a sum up to obtain a collective cross section area of many particles per unit quantum space volume [18]. In fact, such a volume is taken to be responsible for scattering cross-section coefficient variations. Intuitively therefore, geometrical cross sectional area can be used to express scattering cross-section in terms of the geometric cross-section using Equating (1):

$$
\sigma_{\text {geom }}=\pi r^{2}
$$

where $r$ is the particle radius.
When such a particle is assumed to be relatively very small to a level where it is approaching Rayleigh wavelength scattering diameter range, D, then Equating (2) applies:

$$
\frac{\pi D}{\lambda}=\frac{2 \pi r^{2}}{\lambda}=q<<1
$$

This leads to a condition where particle scattering and absorption cross-sections can be generalized using Equating (3) for scattering crosssections:

$$
\begin{aligned}
& \sigma_{s}=\frac{2}{3} \frac{\pi^{5} D^{6}}{\lambda^{4}}|K|^{2}=\frac{2^{7}}{3} \frac{\pi^{5} r^{6}}{\lambda^{4}}|K|^{2}= \\
& =\pi r^{2} \frac{2^{3}}{3} \frac{2^{4} \pi^{4} r^{4}}{\lambda^{4}}|K|^{2}=\frac{8}{3} q^{4}|K|^{2} \sigma_{\text {geom }}
\end{aligned}
$$

and Equating (4) for geometrical cross-sections: 


$$
\begin{aligned}
& \sigma_{a}=\frac{\pi^{2} D^{3}}{\lambda} \operatorname{Im}(-K)= \\
& =\frac{\pi^{2} 2^{3} r^{3}}{\lambda} \operatorname{Im}(-K)= \\
& =\pi r^{2} 2^{3} \frac{\pi r}{\lambda} \operatorname{Im}(-K)= \\
& =4 q \operatorname{Im}(-K) \sigma_{\text {geom }}
\end{aligned}
$$

where $n_{c}$ is the complex index of refraction and $K$ can be expressed as Equating (5):

$$
K=\left(n_{c}^{2-1}\right) /\left(n_{c}^{2}+2\right) .
$$

It is therefore acceptable that the real index of refraction of the media affects the speed of the radiation while the imaginary index of refraction affects the amplitude of the light radiation. Thus, the total extinction cross-section, $\sigma_{e}$, becomes the sum of the absorption and scattering cross-sections expressed in Equating (6) as:

$$
\sigma_{e}=\sigma_{a}+\sigma_{s}
$$

Such a simple approach explains the several characteristics exhibited by wide-band a gap optoelectronic material that makes them more useful for many applications as compared to lower band gap optoelectronic materials limited to light absorbing applications. Its believed that a device incorporating such a feature exhibit switchable larger voltage capabilities [6] and brings its electronic transition energies within the range of the energy of visible light spectra. A system can be modeled to exhibit superposition of two independent and distinguishable quantum states [6]. Analyzing such a two-level quantum system becomes imperative to use the Hilbert space to describe it exhaustively. This model has its model space anchored on a two independent states system for its simplicity as a quantum system as it adopts simple linear differential equations within linear algebra two-dimensional space [18].

\section{MODEL DEVELOPMENT}

A small segment of medium is chosen and considered as having a refractive index, $\eta$, and crosssectional area, $\delta A$, between $z$, and, $z+\delta z$ for inci- dent light intensity, $I(z)$ which can be integrated over all frequencies of the specified chosen spectra. For purposes of this model, propagation is assume to take place in one direction and ignores any non-directional spontaneous emission and model using Figure 1.

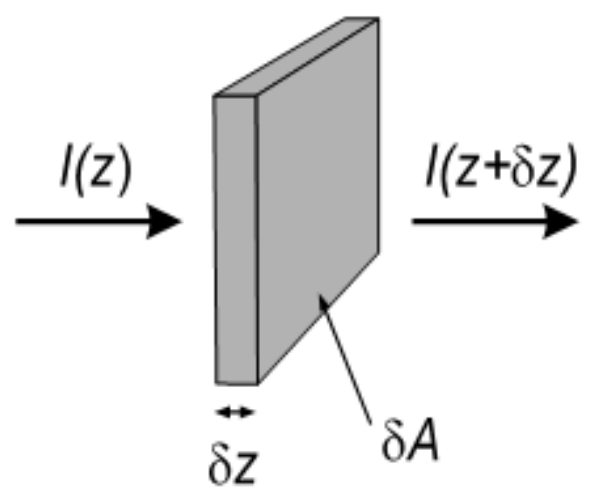

Figure 1 - Model Dimensions

The population densities in the left and right states as depicted by figure 1 are equated as $n_{\mathrm{m}}$ and $n_{\mathrm{n}}$ to represent lower and upper states respectively so that the total change in power on propagating through the model figure 1 segment can be expressed as in Equating (7):

$$
(I(z)-I(z+\delta z)) \delta A=-\frac{\partial(z)}{\partial z} \delta z \delta A
$$

or in terms of the radiation density, $U$ and Einstein, $B$, coefficients as in Equating (8):

$$
-\frac{\partial(z)}{\partial z} \delta z \delta A=B U(v, z) h v\left(n_{m}-n_{n}\right) \delta z \delta A
$$

A laser ion that can be excited into a higher energy level and may spontaneously return to its ground state by releasing the acquired energy in the form of a photon [1]. Such stimulated emission is as a result of vacuum noise. In this model a laser ion is placed in a microcavity structure which will undergo modifications in structure under an optical field as depicted by Figure 2. In quantum level mechanics, an energy level is degenerate only if it corresponds to either two or more different measurable states of a quantum system or if they give the same value of energy upon measurement which correspond to a particular energy level $[17,2]$. 
Based on a spectral line profile function depicted in Figure 2, intensity at a particular frequency, $v$, can be expressed as using Equating (9):

$$
I(z, v)=I(z) f(v),
$$

where $f(v)$ is the spectral line profile function.

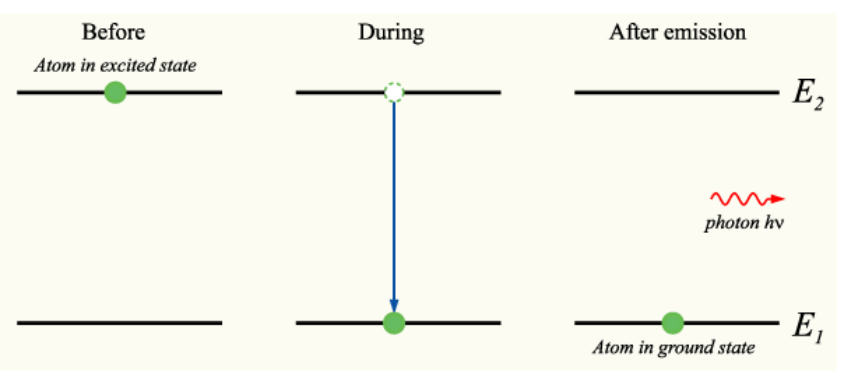

Figure 2 - Model of spontaneous emission

Integrating Equating (9) we obtain Equating (10), where we have at orthogonal conditions:

$$
\int_{0}^{\infty} f(v) d v=1
$$

This implies that the intensity can be related to radiation density as Equating (11):

$$
I(z) f(v)=\frac{c}{\eta} U(v, z) .
$$

Substituting, $U$, the rate of loss of intensity can be expressed as Equating (12):

$$
\frac{\partial I(z)}{\partial z}=-\frac{\eta B I(z) f(v) h v\left(n_{1}-n_{2}\right)}{c} .
$$

The function takes the form, Equating (13):

$$
\frac{d y}{d x}=-k y
$$

with its solution given in Equating (14):

$$
y=y_{0} e^{-k x}
$$

Hence, we can identify an absorption coefficient, $K$, as in Equating (15):

$$
k(v)=\frac{\eta B f(v) h v\left(n_{1}-n_{2}\right)}{c} .
$$

which is a mathematically representative of a Hamiltonian space with the same energy eigenvalue for a system having more than one linearly independent eigenstates.

Taking into account the degeneracy of these states, in relationship to $A$ and $B$ coefficients, the relationship can be written as in Equating (16):

$$
\begin{aligned}
& k(v)=\frac{c^{2} A}{8 \pi v^{2} \eta^{2}} \cdot \frac{g_{n}}{g_{m}}\left(n_{m}-\frac{g_{m}}{g_{n}} n_{n}\right) f(v)= \\
& =\mathrm{K}(v) \cdot\left(n_{m}-\frac{g_{m}}{g_{n}} n_{n}\right) f(v)
\end{aligned}
$$

which is an informal Füchbauer-Ladeberg formula or equation.

Assuming a normal sample of quantum particles under thermal equilibria, the lower state will have overwhelmingly larger population resulting into, $k(v)$ being positive hence absorption while the upper state has $k(v)$ negative and amplification takes place. This defines $k(v)$ as a smallsignal gain coefficient which is a requirement for all laser resonators or laser cavities.

This model considered a laser cavity [4] containing a medium of length $L$ with a gain of $k$ per unit length that mirrors with reflectivities $R_{1}$ and $R_{2}$ as depicted in figure 1 ignoring all other sources of losses. For only one round-trip in the laser cavity, energy gain can be expressed as in Equating (17):

$$
G=R_{1} R_{2} e^{2 k l}=e^{2(k L-\gamma)},
$$

where $R_{1} R_{2}=e^{-2 \gamma}$.

Taking, $k$, is a function of wavelength, and considering only amplified frequencies such that Equating (16) becomes Equating (18):

$$
k(v) L \geq \gamma / L
$$

and using Equating (19):

$$
k(v)=-\mathrm{K}(v) \cdot\left(n_{m}-\frac{g_{m}}{g_{n}} n_{n}\right) f(v)
$$


Then the threshold condition forms a skirt transition band that is summed up by Equating (20):

$$
\left(\frac{g_{m}}{g_{n}} n_{n}-n_{m}\right) \geq \frac{\gamma}{\mathrm{K}(v) f(v) L}
$$

If there is no particle that is excited by absorption, then centre frequency absorption coefficient transition, $v_{0}$ becomes Equating (21):

$$
k_{0}=\mathrm{K}\left(v_{0}\right) f\left(v_{0}\right) n_{0}
$$

where $n_{0}$ is the total number density of atoms. The consequence is that threshold condition simplifies further to Equating (22):

$$
\left(\frac{g_{m}}{g_{n}} n_{n}-n_{m}\right) \geq \frac{n_{0} \gamma}{k_{0} L}
$$

giving a fractional population inversion Equating (23):

$$
n_{p} \geq \gamma / k_{0} L
$$

Extinction coefficient is a significant parameter of a laser $[6,16]$. Its intensity attenuation, $I$, according to our model changes as it passes through the slab model Figure 1 with unit thickness, $d z$ varying with absorption extinction coefficient, $6_{a}$, in inverse length as Equating (24):

$d I=-\alpha_{a} I d z=-I d \tau$

where $\delta \tau$ is the change in the optical depth.

This implies that once light passes through the model slab thickness length, $L$, intensity will vary as in Equating (25:)

$I(L)=I_{0} \mathrm{e}^{-\alpha a L}$

where the intensity of the light entering the slab is $I_{0}$. Therefore it will relate to the extinction coefficient as in Equating (26):

$$
\tau\left(v, z_{1}, z_{2}\right)=\int_{z_{1}}^{z_{2}} \alpha(v, z) d z
$$

The dependence of extinction coefficient along the path has a dependence on frequency [15].
The radiative transfer after passing through a length, $L$, through the model slab, relates to light intensity as in Equating (27):

$$
\begin{aligned}
& I\left(L=z_{2}-z_{1}\right)= \\
& =I_{0} \exp \left[\int_{z_{1}}^{z_{2}} \alpha(v, z) d z\right]= \\
& =I_{0} \exp \left[-\tau\left(v, z_{1}, z_{2}\right)\right]=I_{0} e^{-\tau}
\end{aligned}
$$

\section{RESULTS AND DISCUSSIONS}

Optical confinement is virtually intended to increase gain length of radiation prior to emission from a laser device achieved by increasing that gain path length. This path length depends on the properties of the laser beam in question [13]. Position and curvature of alter and equally optimize laser performance [11] or laser quality factor, $Q$ in terms of frequency and bandwidth as in Equating (29):

$$
Q=\frac{v}{\Delta v}
$$

A laser oscillator is banded between two extreme values. It is built from a trend indicator within overbought or oversold conditions [8, 4, 5]. Any resulting energy loss can be quantified as in Equating (30):

$$
W(t)=W_{0} e^{-\frac{2 \pi v_{0} t}{Q}}
$$

The number of photons remaining in the model cavity at time, $t$, can be given by Eq Equating (31):

$$
P(t)=P_{0} e^{-\frac{\gamma}{\tau} t}
$$

where, $\tau$ is the round-trip time of a photon in the cavity.

The characteristic time for a photon remaining in the cavity can be expressed as in Equating (32):

$$
t_{p}=\frac{\tau}{\gamma}
$$

By comparing the round-trip time of a photon with a quality factor $Q$, we can obtain Equating (33): 


$$
\frac{Q}{2 \pi v_{0}}=\frac{\tau}{\gamma}
$$

or Equating (34):

$$
Q=2 \pi v_{0}\left(\frac{\tau}{\gamma}\right)=2 \pi v_{0} t_{p}
$$

In a nut shell, the bandwidth of the laser [12] is finally simplified by Equating (35):

$$
\Delta v=\frac{1}{2 \pi t_{p}}
$$

\section{MODEL TESTING}

\section{Case One: Using transition for HeNe laser}

Assuming that the lifetime of an upper level transition for certain laser at $632.8 \mathrm{~nm}$ is $1 \times 10^{-7} \mathrm{~s}$, the degree of population inversion that result into a gain coefficient of $0.07 \mathrm{~m}^{-1}$ with its upper and lower states are equal at a refractive index of 1 , then, the line profile function can be obtained from Equating (36) and Equating (37) respectively:

$$
\begin{aligned}
& f(v)=0,\left|v-v_{0}\right|>\Delta v / 2 \\
& f(v)=1 / \Delta v,\left|v-v_{0}\right| \leq \Delta v / 2
\end{aligned}
$$

At a lifetime of $1 \times 10^{-7} \mathrm{~s}$ with an Einstein coefficient of $1 \times 10^{7} \mathrm{~s}^{-1}$ we obtain a frequency of $0.5 \mathrm{THz}, \quad \mathrm{a}$ transition frequency, $f(v)=6.28 \times 10^{-7} \mathrm{~Hz}^{-1}$ and the density of population inversion becomes $7.82 \times 10^{11} \mathrm{~m}^{-3}$ which shows that there must be $7.82 \times 10^{11} \mathrm{~m}^{-3}$ more atoms in their upper state than in the lower.

\section{Case Two: Un-pumped laser cavity}

Supposing that we have an un-pumped laser cavity of $0.5 \mathrm{~m}$ long experiencing a $2 \%$ loss per round trip, then applying this model in Figure 1, we can apply the relation:

$e^{-2 \gamma}=r_{1} r_{2} \Rightarrow \gamma=0.05$, which leads to given: $0.98=e^{-\gamma} \Rightarrow \gamma=0.02$

implying that: $t_{p}=\frac{2 L}{\gamma c}=0.17 \mu \mathrm{s}$

Finally resulting into a bandwidth of:

$$
\Delta v=\frac{1}{2 \pi t_{p}}=0.94 \mathrm{MHz}
$$

\section{Case Three: Threshold condition for Laser action}

In a simple two-level quantum system $[19,10,9]$, at a population inversion condition $N_{n}>N_{m}$ that liberates a strong stimulating field that prevents spontaneous states, a black body mode density, we get Equating (38):

$$
U(v)>>\frac{8 \pi h v^{3}}{c^{3}} \cdot \frac{1}{e^{h v / k T}-1}
$$

Performing best fit and substituting within energy condition $h v$ is $5 \mathrm{eV}$, at a higher temperature of $1000 \mathrm{~K}$ [3] which in essence, approximate $k T$ value to $0.086 \mathrm{eV}$, we obtain (39):

$$
\frac{N_{n}}{N_{m}}=e^{-5 / 0.086}=1.5 \times 10^{-25} .
$$

This shows that a laser media [7] can be simplified as a coherent ensemble of particles or atoms whose bandwidth laser line depends on how its constructed.

\section{CONCLUSION}

A quantum system approach was used to describe and analyze an energy level as a degenerate state that corresponds to different measurable states of a quantum system. Two different degenerate states of a quantum mechanical system were modeled by considering a laser cavity containing a medium of length $L$ with a gain of $k$ per unit length and each constituted by different inhomogeneous broadening Stark levels. A model was developed for a laser device by increasing its gain path length and the result insinuated that a laser medium is a coherent ensemble of particles whose bandwidth laser line depends on how a laser is designed and constructed. 


\section{REFERENCES}

1. Franco, R. L., Bellomo, B., Maniscalco, S., \& Compagno, G. (2013). Dynamics of quantum correlations in two-qubit systems within non-markovian environments. International Journal of Modern Physics B, 27(01n03), 1345053. doi: 10.1142/s0217979213450537

2. He, Y., He, Y.-M., Wei, Y.-J., Jiang, X., Chen, M.-C., Xiong, F.-L., ... Pan, J.-W. (2013). Indistinguishable Tunable Single Photons Emitted by Spin-Flip Raman Transitions in InGaAs Quantum Dots. Physical Review Letters, 111(23). doi: 10.1103/physrevlett.111.237403

3. Hegerfeldt, G. C. (2013). Driving at the Quantum Speed Limit: Optimal Control of a Two-Level System. Physical Review Letters, 111(26). doi: 10.1103/physrevlett.111.260501

4. Hinkley, N., Sherman, J. A., Phillips, N. B., Schioppo, M., Lemke, N. D., Beloy, K., ... Ludlow, A. D. (2013). An Atomic Clock with 10-18 Instability. Science, 341(6151), 1215-1218. doi: 10.1126/science.1240420

5. Höning, M., Muth, D., Petrosyan, D., \& Fleischhauer, M. (2013). Steady-state crystallization of Rydberg excitations in an optically driven lattice gas. Physical Review A, 87(2). doi: 10.1103/physreva.87.023401

6. Kaminskii, A. A. (1990). Laser crystals: their physics and properties (2nd ed.). Berlin: Springer.

7. Knight, P. L., \& Radmore, P. M. (1982). Quantum revivals of a two-level system driven by chaotic radiation. Physics Letters A, 90(7), 342-346. doi: 10.1016/0375-9601(82)90625-9

8. Koechner, W. (2006). Solid-state laser engineering. New York: Springer.

9. Lu, X.-J., Chen, X., Ruschhaupt, A., Alonso, D., Guérin, S., \& Muga, J. G. (2013). Fast and robust population transfer in two-level quantum systems with dephasing noise and/or systematic frequency errors. Physical Review A, 88(3), 033406. doi: 10.1103/physreva.88.033406

10. Martin, M. J., Bishof, M., Swallows, M. D., Zhang, X., Benko, C., von-Stecher, J., ... Ye, J. (2013). A Quantum Many-Body Spin System in an Optical Lattice Clock. Science, 341(6146), 632-636. doi: 10.1126/science.1236929

11. Meystre, P., \& Sargent, M. (2010). Elements of quantum optics (4th ed.). Berlin: Springer.

12. Mosiori, C. O. (2016). Effects of Quantum Confinements in Tin Sulphide Nanocrystals Produced by Wet-Solution Technique. Asia Pacific Journal of Energy and Environment, 3(2), 73-80.

13. Mosiori, C. O., Kwembur, M., \& Maera, J. (2016). Thermal Emittance and Solar Absorptance of CdS Thin Films. International Journal of Engineering Inventions, 4(11), 01-05.

14. Mosiori, C. O., Maera, J., Njoroge, W., Shikambe, T., Munji, M., \& Magare, R. (2015). Modeling Transfer of electrons between Energy States of an Electrolyte and CdS thin films using Gerischer Model. Engineering International, 3(1), 35-44.

15. Ostermann, L., Ritsch, H., \& Genes, C. (2013). Protected State Enhanced Quantum Metrology with Interacting Two-Level Ensembles. Physical Review Letters, 111(12), 123601. doi: 10.1103/physrevlett.111.123601

16. Panock, R., \& Temkin, R. (1977). Interaction of two laser fields with a three-level molecular system. IEEE Journal of Quantum Electronics, 13(6), 425-434. doi: 10.1109/jqe.1977.1069354

17. Patel, R. B., Bennett, A. J., Farrer, I., Nicoll, C. A., Ritchie, D. A., \& Shields, A. J. (2010). Two-photon interference of the emission from electrically tunable remote quantum dots. Nature Photonics, 4(9), 632-635. doi: 10.1038/nphoton.2010.161

18. Polley, E. H., Apple, D. J., \& Bizzell, J. W. (1975). The laser as a research tool in visual system investigation. American Psychologist, 30(3), 340-348. doi: 10.1037/0003-066x.30.3.340

19. Ruschhaupt, A., Chen, X., Alonso, D., \& Muga, J. G. (2012). Optimally robust shortcuts to population inversion in two-level quantum systems. New Journal of Physics, 14(9), 093040. doi: 10.1088/1367-2630/14/9/093040 
20. Yeo, I., de Assis, P.-L., Gloppe, A., Dupont-Ferrier, E., Verlot, P., Malik, N. S., ... Richard, M. (2013). Strain-mediated coupling in a quantum dot-mechanical oscillator hybrid system. Nature Nanotechnology, 9(2), 106-110. doi: 10.1038/nnano.2013.274 